\title{
Different degrees of lunar synchronization of ovary development between two morphs of a Tanganyikan cichlid fish
}

\author{
AUTHOR(S): \\ Takahashi, Tetsumi
}

\section{CITATION:}

Takahashi, Tetsumi. Different degrees of lunar synchronization of ovary development between two morphs of a Tanganyikan cichlid fish. Hydrobiologia 2010, 644(1): 139-143

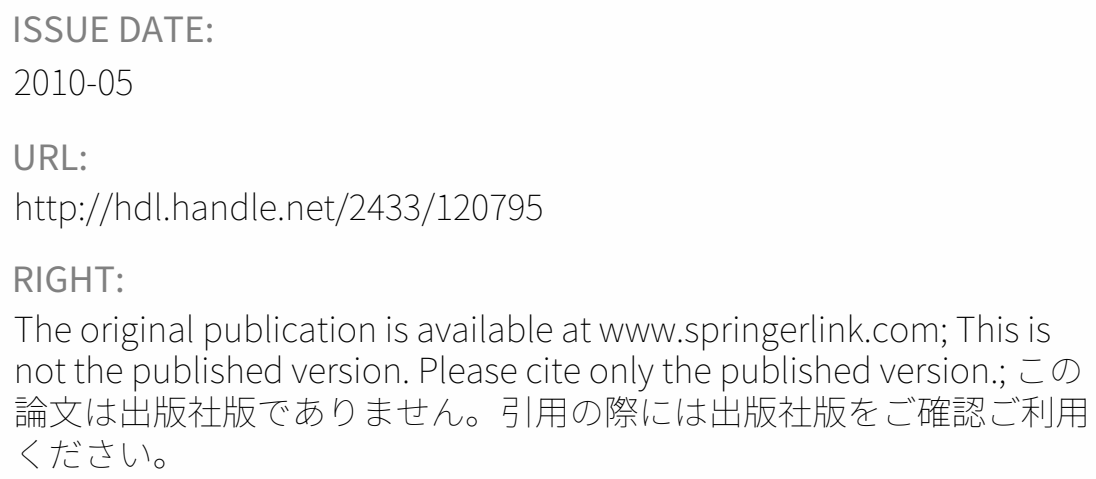


1 Different degrees of lunar synchronization of ovary development between two

2 morphs of a Tanganyikan cichlid fish

3

4 Tetsumi Takahashi

5 Graduate School of Science, Kyoto University, Kitashirakawa-Oiwake, Sakyo, Kyoto

6 606-8502, Japan

7 E-mail: tetsumi@terra.zool.kyoto-u.ac.jp

8

9 Key words: Cichlidae; gonado-somatic index; lunar cyclic spawning; spawning nest;

10 predator avoidance strategy

11

12 Two gray-scale figures, no tables, no appendix.

13

14 This paper has not been submitted elsewhere in identical or similar form, nor will it be

15 during the first three months after its submission to Hydrobiologia. 
16 Abstract The degree of lunar synchronization of spawning is thought to be related to

17 a strategy that decreases predation on the brood in Tanganyikan substrate-brooding

18 cichlids. Here, I examined the periodic change of ovary development in two morphs of

19 Telmatochromis temporalis: the normal morph uses burrows under stones as spawning

20 nests, whereas the dwarf morph uses holes within empty snail shells. The normal morph

21 showed a significant lunar synchronization of ovary development, but the dwarf morph

22 did not. In the normal morph, spawning prior to the full moon probably decreases the

23 incidence of approaching brood predators and increases the guarding efficiency of

24 parents. In the dwarf morph, however, lunar cyclic spawning may be dispensable,

25 because the spawning nests within shells are probably highly effective for predator

26 avoidance. These two morphs are closely related, and then will be a good model to

27 clarify the widely observed phenomenon of lunar cyclic spawning. 


\section{Introduction}

30 Although biological rhythmicity that is synchronized with lunar or semi-lunar cycles is

31 a widespread phenomenon in marine organisms (Leatherland et al., 1992; Hernández-

32 León, 2008), it is rare among organisms inhabiting freshwater bodies, where tidal

33 fluctuation is limited or absent. However, lunar cyclic spawning has been reported in

34 nine substrate-brooding species of cichlid fish belonging to the tribe Lamprlogini in

35 Lake Tanganyika, Africa (Nakai et al., 1990; Rossiter, 1991). In these species, a pair of

36 parents or a female guards the eggs and yolk-sac larvae, which cannot easily escape

37 from predators by themselves. The spawning cycles of these species are thought to

38 improve the survival of the vulnerable brood (eggs and yolk-sac larvae) or dispersing

39 young (Nakai et al., 1990; Rossiter, 1991). Three possible explanations have been

40 proposed: 1) spawning prior to the full moon reduces the predation on the vulnerable

41 brood by nocturnal predators (bagrid catfish) that are not active during the full moon

42 (Rossiter, 1991), 2) spawning prior to the full moon enhances the effectiveness of

43 nocturnal parental guarding of the vulnerable brood under the maximum lunar

44 illumination during the full moon (Nakai et al., 1990; Rossiter, 1991), and 3) dispersal

45 of young during the fourth quarter of the lunar cycle and the new moon improves the

46 survival of young dispersing under the cover of darkness (Nakai et al., 1990). If any of

47 these explanations is true, the degree of lunar synchronization of spawning will vary

48 according to the strategies for decreasing predation on the vulnerable brood and/or

49 dispersing young. Comparison between more closely related populations is better to test

50 this hypothesis because it will minimize the effect of phylogeny. 
is an iteroparous substrate brooder. Like other substrate-brooding species that are known to exhibit lunar synchronized spawning, this fish also belongs to the tribe

Lamprologini (Takahashi, 2003). T. temporalis is dimorphic for body size. The two morphs dwell in different habitats, lay eggs and guard the brood in different types of nest, and hide in different types of shelter, respectively (Takahashi, 2004; Takahashi et al., 2009). The normal morph possesses a moderate-sized body for a Tanganyikan rockdwelling cichlid $\left[88 \mathrm{~mm}\right.$ in standard length at maximum size $\left(\mathrm{SL}_{\max }\right)$ in males, $62 \mathrm{~mm}$ $\mathrm{SL}_{\max }$ in females] and is one of the most common fish on rocky shorelines. This morph uses burrows under stones as spawning nests and shelters (Mboko \& Kohda, 1999;

61 Katoh et al., 2005). The dwarf morph is about half of the normal morph in body size (45 mm SL $\mathrm{max}_{\max }$ in males, $29 \mathrm{~mm} \mathrm{SL}_{\max }$ in females) and invariably inhabits shell beds, in which the lake bottom is covered by a high density of empty snail shells of the gastropod Neothauma tanganyicense Smith (Takahashi et al., 2009: fig. 1d). This morph uses the empty shells as spawning nests and shelters. A population genetic study showed that the normal and dwarf morphs from Wonzye $\left(08^{\circ} 43^{\prime} 31^{\prime \prime} \mathrm{S} ; 31^{\circ} 07^{\prime} 55^{\prime \prime} \mathrm{E}\right.$; near Mpulungu, Zambia, at the southern end of the lake) were closely related but isolated from each other, although their geographical distributions partly overlap (Takahashi et al., 2009).

In the present study, I observed the periodic changes of ovary development in the normal and dwarf morphs from Wonzye, and examined whether the ovary development was synchronized with the lunar cycle. Based on my findings, I discuss the relationship between the degree of lunar synchronization of ovary development and 74 predator avoidance strategies. 
Materials and Methods

Sampling of fish

80

81 Using SCUBA diving, 6 to 11 females of the normal morph were collected every 3 to 5 days between 27 September and 2 December of 2005 on rocky shorelines at 1.0 to 4.9 m depth $(N=173)$, and 5 to 12 females of the dwarf morph were collected every 3 or 4 days between 29 September and 1 December of 2005 on shell beds at 9.5 to $10.3 \mathrm{~m}$ depth $(N=174)$. Fish were transported to the laboratory at Mpulungu and killed in a solution of anesthesia FA 100 (Takeda Pharmaceutical Co. Ltd.) within 6 hours after collection. The ovaries were extracted from all females. The bodies and ovaries were dabbed on tissue paper to remove excess moisture before weighing, and were weighed to the nearest $1 \mathrm{mg}$. When the ovary was lighter than the minimum readable weight of the electronic balance $(1 \mathrm{mg})$, the weight was taken to be $0 \mathrm{mg}$. However, zero values cannot be subjected to log-transformation. To avoid this problem, I added $1 \mathrm{mg}$ to all ovary weights. The gonado-somatic index was calculated as $I_{\mathrm{G}}=100 W_{\mathrm{O}} W_{\mathrm{B}}^{-1}(\%)$, where $W_{\mathrm{B}}$ is body weight $(\mathrm{mg})$, and $W_{\mathrm{O}}$ is ovary weight $+1(\mathrm{mg})$. Note that $I_{\mathrm{G}}$ is not a useful descriptor of gonad investment in some organisms (Tomkins and Simmons, 2002). However, this index was used in the present study, because the normal and dwarf morphs showed isometric relationships between $W_{\mathrm{B}}$ and $W_{\mathrm{O}}$ (see below in Results). 
$100 I_{\mathrm{G}}$ was approximated to a cosine curve:

101

102

$f\left(T_{i}\right)=a \cos \left[2 \pi\left(T_{i}-x\right) / l\right]+y$

$103\left[f\left(T_{i}\right) \geq 0,0 \leq x<l\right]$,

104

105

where $T_{i}$ is the number of days from 27 September (the first day of sampling) to the day

that individual $i$ was collected, $a, x$ and $l$ are amplitude, phase and length of the cosine

curve, respectively, and $y$ is a corrected average of $I_{\mathrm{G}}$. Appropriate values for parameters

were searched by the method of least squares in three models: full model (four

109

parameters: $a, x, l$ and $y$ ), lunar-cyclic model with $l=29.5$ (three parameters: $a, x$ and $y$ )

111 these three models for each morph.

\section{Results}

116 Body weight and ovary weight

118 The relationship between $W_{\mathrm{B}}$ and $W_{\mathrm{O}}$ is shown in Fig. 1. In the normal morph, all small

119 females lighter than $1000 \mathrm{mg}$ possessed light ovaries. These small females were

120 considered to be immature and were excluded from the following analyses. Some large

121 females heavier than $1000 \mathrm{mg}$ also had light ovaries. However, these females were

122 considered to be mature females having undeveloped ovaries, for example, females just

123 after spawning, and were not excluded from the following analyses. In the dwarf morph, 
124 all females were likely mature, although their $W_{\mathrm{B}}$ was much lighter than that of the mature females of the normal morph. The average $W_{\mathrm{B}}$ of the mature females was 2050 $\mathrm{mg}(N=160)$ in the normal morph and $259 \mathrm{mg}(N=174)$ in the dwarf morph. Tomkins and Simons (2002) pointed out that $I_{\mathrm{G}}$ was not a useful descriptor of gonad investment in organisms that showed an allometric relationship between the gonad and somatic weights. In the present study, however, the isometric function provided a significantly better fit than the allometric function for explaining the

131 relationship between $W_{\mathrm{B}}$ and $W_{\mathrm{O}}$ in each morph (Fig. 1) (test of difference between

132 allometric and isometric functions: $F=0.483, \mathrm{df}=1$ and $158, P=0.488$ in the normal

133 morph; $F=0.034, \mathrm{df}=1$ and $172, P=0.854$ in the dwarf morph). This result means that

$134 I_{\mathrm{G}}$ is not significantly affected by $W_{\mathrm{B}}$ after maturity; therefore, $I_{\mathrm{G}}$ was used as the

135 descriptor of gonad investment in the present study. In the normal morph, the variance

136 of $\log \left(W_{\mathrm{O}}\right)$ seemed to increase with $\log \left(W_{\mathrm{B}}\right)$ (Fig. 1), but this tendency was not

137 significant [the correlation coefficient between $\log \left(W_{\mathrm{B}}\right)$ and squared deviates was $r=$ $138 \quad 0.071, P=0.370]$.

140 Lunar synchronization of ovary development

142 In mature females of the normal morph, $I_{\mathrm{G}}$ showed a marginally significant difference among sampling days (Kruskal-Wallis: $\chi^{2}=33.3$, df $=19, P=0.022$ ) and a significant

144 fit to the lunar-cyclic model (Fig. 2A) [full model versus lunar-cyclic model: $F=0.819$, $145 \mathrm{df}=1$ and 156, $P=0.367$; lunar-cyclic model versus non-cyclic model: $F=6.32, \mathrm{df}=2$ 146 and $157, P=0.002$; this result was also supported by analyses using $\left.\log \left(I_{\mathrm{G}}\right)\right] . I_{\mathrm{G}}$ peaked 147 prior to the full moon (13 days). In the dwarf morph, some females collected during the 
148 second quarter of the lunar cycle (7-15 days) possessed higher $I_{\mathrm{G}}$ than females collected

149 during the fourth quarter (22-0 days), like the normal morph (Fig. 2B). However, the

150 difference of $I_{\mathrm{G}}$ among sampling days was not significant (Kruskal-Wallis: $\chi^{2}=15.6$, df

$151=18, P=0.618)$ and $I_{\mathrm{G}}$ did not significantly fit the full and lunar-cyclic models [full

152 model versus lunar-cyclic model: $F=1.81, \mathrm{df}=1$ and $170, P=0.180$; lunar-cyclic

153 model versus non-cyclic model: $F=2.38, \mathrm{df}=2$ and $171, P=0.096$; this result was also

154 supported by analyses using $\left.\log \left(I_{\mathrm{G}}\right)\right]$. This result suggests that the ovary development of

155 the dwarf morph was not synchronized with the lunar cycle, or that the degree of lunar

156 synchronization of ovary development was lower than the detection level of the present 157 test.

160 Discussion

The present study revealed that 1) the ovary development of the normal morph was significantly synchronized with the lunar cycle, and 2) the ovary development of the

164 dwarf morph was not significantly synchronized with the lunar cycle $(P=0.096)$, or at

165 least the cycle was less pronounced compared to the normal morph. The ovary

166 development of the normal morph peaked during the second quarter of the lunar cycle,

167 suggesting high activity of reproduction during this period, in accord with the findings

168 for nine other substrate-brooding species of Tanganyikan cichlids (Nakai et al., 1990;

169 Rossiter, 1991). Three possible explanations have been proposed for the lunar cyclic

170 spawning of the Tanganyikan substrate brooders, and two of these explanations are

171 applicable to the normal morph. 
173 the brood (egg and yolk-sac stages) with a period when nocturnal predators are not

174 active (Rossiter, 1991). The bagrid catfish is one of the main predators in the lake (Fryer

175 and Iles, 1972). Small bagrid catfishes (Phyllonemus spp. and young of Chrysichthys

176 spp.) are ubiquitous in rocky shorelines, which are the main habitat of the normal morph.

177 Predation by these catfishes will critically affect the survival of the broods of the normal

178 morph. Young of the normal morph hatch out about 3 days after spawning (Katoh et al.,

179 2005), and therefore the vulnerable stages of the brood occur around the full moon,

180 which accords with a period when bagrid catfishes are not active (McKay, 1983;

181 Rossiter, 1991).

The second applicable explanation is synchronization of the vulnerable stages

of the brood with a period when the nocturnal parental guarding is effective (Nakai et al.,

184 1990; Rossiter, 1991). The parents of the normal morph attack and repel the brood

185 predators when they approach (Mboko and Kohda, 1999). The ambient light during the full moon may assist the parents in visual detection at night, and in repelling approaching nocturnal brood predators, for example, spiny eels (Ochi et al., 1999). The other proposed explanation for lunar cyclic spawning, namely, that

189 spawning prior to the full moon improves the survival of young dispersing under the

190 cover of darkness (Nakai et al., 1990), is unlikely in the normal morph. The young of some substrate-brooding species leave the spawning nest immediately after they complete yolk absorption. The period of yolk absorption is about 2 weeks (Kuwamura,

193 1997), resulting in the dispersal of the young during dark nights during the fourth

194 quarter and new moon (Nakai et al., 1990). However, the young of the normal morph

195 remain in the spawning nest for more than 1 month after yolk absorption (Mboko and 
196 Kohda, 1999). The timing of dispersal of the free-swimming young of the normal

197 morph may be decided by some environmental cue.

198 As discussed above, the lunar cyclic spawning of the normal morph may

199 improve the survival of the brood by synchronizing the vulnerable stages of the brood

200 with the period when the nocturnal brood predators (bagrid catfish) are not active and

201 the nocturnal parental guarding is effective. On the other hand, the dwarf morph did not

202 show clear lunar synchronization of ovary development. The dwarf morph uses empty

203 snail shells as spawning nests in shell beds. The eggs and yolk-sac larvae were always

204 found with a female close to the end of the hole within a shell $(N=12$, observed in

205 November of 2005 and October to November of 2007 by the author), suggesting that

206 females spawn and care for the brood there. The end of the hole is very small and is

207 invisible from the outside. The predators would probably have trouble finding and

208 accessing the brood. Spawning nests within empty shells, therefore, will be very

209 effective for preventing predation on the vulnerable brood of the dwarf morph, and

210 therefore lunar cyclic spawning may be dispensable for predator avoidance. Release

211 from the limitation of the spawning timing may reduce the degree of lunar

212 synchronization of the reproduction of the dwarf morph.

213 Different degrees of lunar synchronization in spawning have been reported

214 among eight species of Tanganyikan substrate-brooding cichlids, and these variations

215 were suggested to be related to the spawning sites (Nakai et al., 1990). The present

216 statistical test of the lunar synchronization using a cosine-curve function supports this

217 suggestion. However, this explanation for the different degrees of lunar synchronization

218 is based on circumstantial evidence, and other explanations remain possible. In fact,

219 females of Lamprologus callipterus and Altolamprologus compressiceps use shells as 
220

221

222

223

224

225

226

227

228

229

230

231

232

233

234

235

236

237

238

239

240

241

242

243

spawning sites like the dwarf morph of T. temporalis, but show lunar spawning (Nakai et al., 1990). Other factors, such as spawning position within the shells, may also play a role. The present study was conducted from the end of dry season to the beginning of rainy season covering two lunar cycles. More analyses of other life-history traits and replicates in another season will be needed to reveal the mechanism and the adaptive significance of the lunar synchronization.

Lunar cyclic spawning is a well-documented feature in marine organisms (Leatherland et al., 1992; Hernández-León, 2008). More detailed analyses of the normal and dwarf morphs of T. temporalis will help to clarify the mechanism and evolution of this phenomenon.

Acknowledgments I express my sincere thanks to M. Hori for providing facilities for research; to H. Phiri, D. Sinyinza and other staff of the Lake Tanganyika Research Unit in Mpulungu for their full cooperation; to E. Nakajima and D. Sinyinza for comments on the manuscript; to T. Sota, S. Takeyama and K. Ota for advice on research; and F. Tashiro for assistance. This study was carried out in accordance with Guidelines for Animal Experimentation, Kyoto University. This study was supported by Grants-in-Aid for JSPS Fellows (No. 20188), for Special Purposes (No. 18779002) and for Young Scientists (No. 20770065), and Global COE Program (A06), MEXT, Japan.

\section{References}

\section{Fryer, G. \& T. D. Iles, 1972. The cichlid fishes of the Great Lakes of Africa: their} biology and evolution. Oliver \& Boyd, Edinburgh. 
244 Hernández-León, S., 2008. Natural variability of fisheries and lunar illumination: a 245 hypothesis. Fish and Fisheries 9: 138-154.

246 Katoh, R., H. Munehara \& M. Kohda, 2005. Alternative male mating tactics of the 247 substrate brooding cichlid Telmatochromis temporalis in Lake Tanganyika. $248 \quad$ Zoological Science 22: 555-561.

249 Kuwamura, T., 1997. The evolution of parental care and mating systems among 250 Tanganyikan cichlids. In Kawanabe, H., M. Hori \& M. Nagoshi (eds), Fish Communities in Lake Tanganyika. Kyoto University Press, Kyoto: 57-86.

Leatherland, J. F., K. J. Farbridge \& T. Boujard, 1992. Lunar and semi-lunar rhythms in fishes. In Ali, M. A. (ed), Rhythms in Fishes. Plenum Press, New York: 83-107.

Mboko, S. K. \& M. Kohda, 1999. Piracy mating by large males in a monogamous substrate-breeding cichlid in Lake Tanganyika. Journal of Ethology 17: 51-55.

McKaye, K. R., 1983. Ecology and breeding behavior of a cichlid fish, Cyrtocara eucinostomus, on a large lek in Lake Malawi, Africa. Environmental Biology of Fishes 8: 81-96.

Nakai, K., Y. Yanagisawa, T. Sato, Y. Niimura \& M. M. Gashagaza, 1990. Lunar synchronization of spawning in cichlid fishes of the tribe Lamprologini in Lake Tanganyika. Journal of Fish Biology 37: 589-598.

Ochi, H., Y. Sato \& Y. Yanagisawa, 1999. Obligate feeding of cichlid eggs by Caecomastacembelus zebratus in Lake Tanganyika. Journal of Fish Biology 54: $450-459$.

Rossiter, A., 1991. Lunar spawning synchroneity in a freshwater fish. Naturwissenschaften 78: 182-184.

267 Takahashi, T., 2003. Systematics of Tanganyikan cichlid fishes (Teleostei: Perciformes). 
$268 \quad$ Ichthyological Research 50: 367-382.

269 Takahashi, T., 2004. Morphological and genetic distinctness of rock and shell-bed

270 dwelling Telmatochromis (Teleostei, Cichlidae) in the South of Lake Tanganyika

271 suggest the existence of two species. Journal of Fish Biology 65: 419-435.

272 Takahashi, T., K. Watanabe, H. Munehara, L. Rüber \& M. Hori, 2009. Evidence for

273 divergent natural selection of a Lake Tanganyika cichlid inferred from repeated

274 radiations in body size. Molecular Ecology 18: 3110-3119.

275 Tomkins, J. L. \& L. W. Simmons, 2002. Measuring relative investment: a case study of

276 testes investment in species with alternative male reproductive tactics. Animal

277 Behaviour 63: 1009-1016. 


\section{Figure legends}

279

280 Fig. 1 Relationship between the ovary weight and body weight in the normal (solid

281 circles; $N=173$ ) and dwarf (open circles; $N=174$ ) morphs. Regression lines and

282 functions in the graph are of the mature individuals of the normal $(N=160)$ and dwarf

$283(N=174)$ morphs $($ see text $)$

284

285 Fig. 2 Periodic change of ovary development during the study period. The normal

286 morph showed a significant lunar cyclic pattern $(\mathbf{A} ; N=160)$, whereas non-significant

287 cyclic pattern was seen in the dwarf morph $(\mathbf{B} ; N=174)$ (see text). A line in the graph

288 and a function above the graph are of the selected model in each morph 


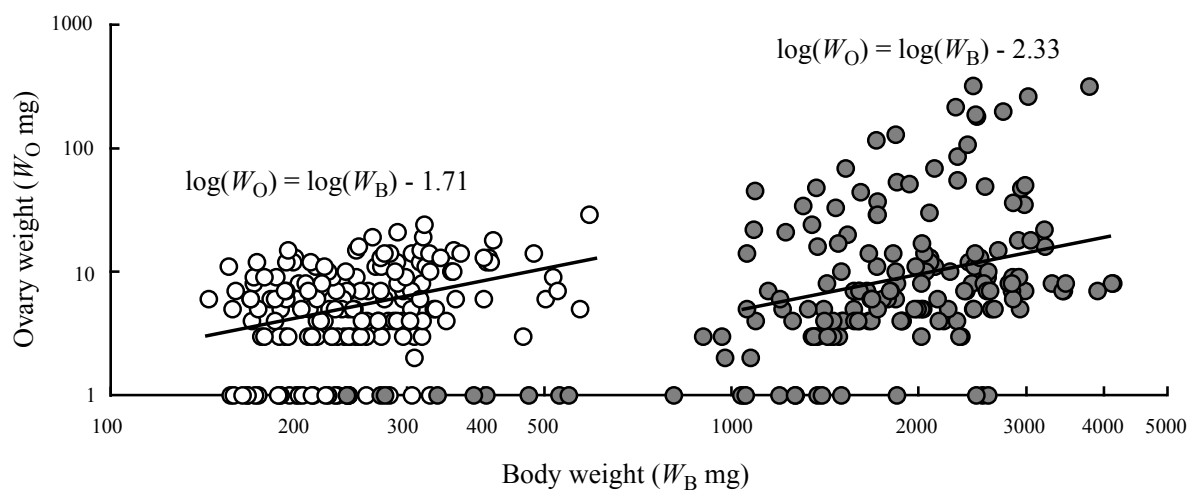


A

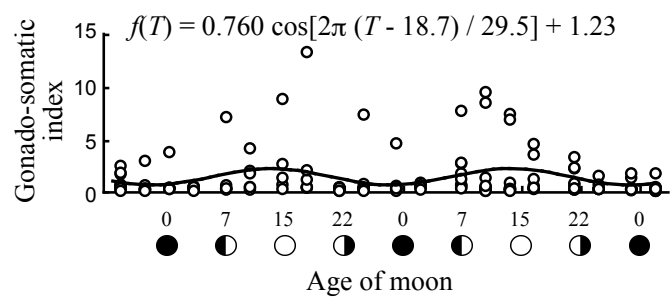

B

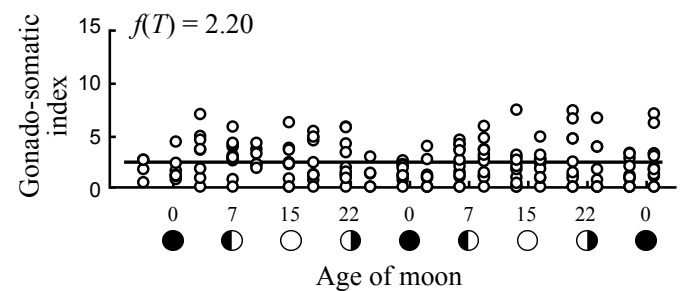

